\title{
Optical counterparts of cosmological GRBs due to heating of ISM in the parent galaxy
}

\author{
G.S. Bisnovatyi-Kogan ${ }^{1}$ and A.N. Timokhin ${ }^{1,2}$ \\ 1 Space Research Institute, Profsoyuznaya 84/32, 117810 Moscow, Russia \\ e-mail: gkogan@mx.iki.rssi.ru \\ 2 Sternberg Astronomical Institute, Universitetskij pr. 13, 119899 Moscow, Russia \\ e-mail: timokhin@mx.iki.rssi.ru
}

Received January 21; accepted July 5, 1999

\begin{abstract}
We investigated influence of cosmological GRB on the surrounding interstellar medium. It was shown that $\gamma$-radiation from the burst heats interstellar gas to the temperatures $>10^{4} \mathrm{~K}$ up to the distance $\sim 10 \mathrm{pc}$. For high density ISM, optical and UV radiation of the heated, gas can be observed on the Earth several years as a GRB's counterpart.
\end{abstract}

Key words: gamma-rays: bursts

\section{Introduction}

In the cosmological model for Gamma-Ray Bursts, energy production in $\gamma$-region must be enormously large, $10^{51}-10^{53}$ ergs, during several seconds. Such huge energy flux interacts with the matter in the parent galaxy leading to its heating and formation of counterparts in another spectral region. We investigate results of this interaction without specifying the mechanism of GRB formation.

The problem is examined by studying the response of interstellar medium with normal chemical composition on propagation through it of a short powerful gamma-ray pulse. Spherical symmetry was accepted; different densities of interstellar gas and burst energies were considered.

\section{Basic equations}

The basic equations, describing this problem are:

$\frac{\partial \varrho}{\partial t}+\frac{1}{r^{2}} \frac{\partial}{\partial r}\left(r^{2} \varrho u\right)=0$,

$\frac{\partial u}{\partial t}+u \frac{\partial u}{\partial r}=-\frac{1}{\varrho} \frac{\partial p}{\partial r}+F_{\gamma}$

$\frac{\partial \epsilon}{\partial t}+u \frac{\partial \epsilon}{\partial r}=-\frac{p}{\varrho} \frac{1}{r^{2}} \frac{\partial}{\partial r}\left(r^{2} u\right)+H_{\gamma}-C_{\gamma}$.

Send offprint requests to: A.N. Timokhin
Where

$p=\left(n_{\mathrm{i}}+n_{\mathrm{n}}+n_{\mathrm{e}}\right) k T, \quad \epsilon=\frac{3}{2} \frac{k T}{\mu \mathrm{m}_{\mathrm{u}}}$,

$n_{\mathrm{n}}, n_{\mathrm{i}}, n_{\mathrm{e}}$ are concentrations of neutral atoms, ions and electrons correspondently.

For the $\gamma$-ray signal interacting with matter due to Thomson scattering of photons on electrons with crosssection $\sigma_{\mathrm{T}}$ we have:

$F_{\gamma}=\frac{1}{c} \frac{L}{4 \pi r^{2}} \frac{\mu_{\mathrm{e}} \sigma_{\mathrm{T}}}{m_{\mathrm{u}}}$.

For $\gamma$-rays with $h \nu \gg B_{\mathrm{e}}^{(a, i)}$ ( $B_{\mathrm{e}}$ is the binding energy of electrons in atoms or ions) the cross-section is almost the same for free and bound electrons. Heating of the gas by the light signal with the spectrum $\frac{\mathrm{d} L}{\mathrm{~d} E}=\frac{L}{E_{\max }} \mathrm{e}^{-E / E_{\max }}$ is given by (Bisnovatyi-Kogan \& Blinnikov 1980):

$H_{\gamma}=\frac{L}{4 \pi r^{2}} \frac{\mu_{\mathrm{e}} \sigma_{\mathrm{T}}}{m_{\mathrm{u}}} \frac{E_{\max }-4 k T}{m_{\mathrm{e}} c^{2}}$,

where $L$ is the energy flux of the signal; $E$ is the energy of photons. This formula is valid for $E \ll m_{\mathrm{e}} c^{2}$. For photons with energies $<10 \mathrm{keV}$, the main process of interaction with the gas is photoabsorption by ions of heavy elements. To take in to account gas heating by soft X-rays photons we enhance term (6) using $E_{\max } \sim 2 \mathrm{MeV}$. Because of a lack of information about soft X-ray spectra of GRBs and variations in interstellar media density such assumption, seems to be quite good. On the other hand, completely ignoring gas heating by X-rays we get a counterpart by less then $2^{\mathrm{m}}$ fainter. Cooling of optically thin plasma by freefree and free-bound transitions is given by approximating function (Cowie et al. 1981):

$C_{\gamma}=\frac{\Lambda(T) n^{2}}{\varrho}, \quad n=n_{\mathrm{n}}+n_{\mathrm{i}}$

$\Lambda(T)= \begin{cases}0, & \text { when } T<10^{4} \mathrm{~K} \\ 1.010^{-24} \cdot T^{0.55}, & \text { when } 10^{4} \mathrm{~K}<T<10^{5} \mathrm{~K} \\ 6.210^{-19} \cdot T^{-0.6}, & \text { when } 10^{5} \mathrm{~K}<T<410^{7} \mathrm{~K} \\ 2.510^{-27} \cdot T^{0.5}, & \text { when } T>410^{7} \mathrm{~K}\end{cases}$ 

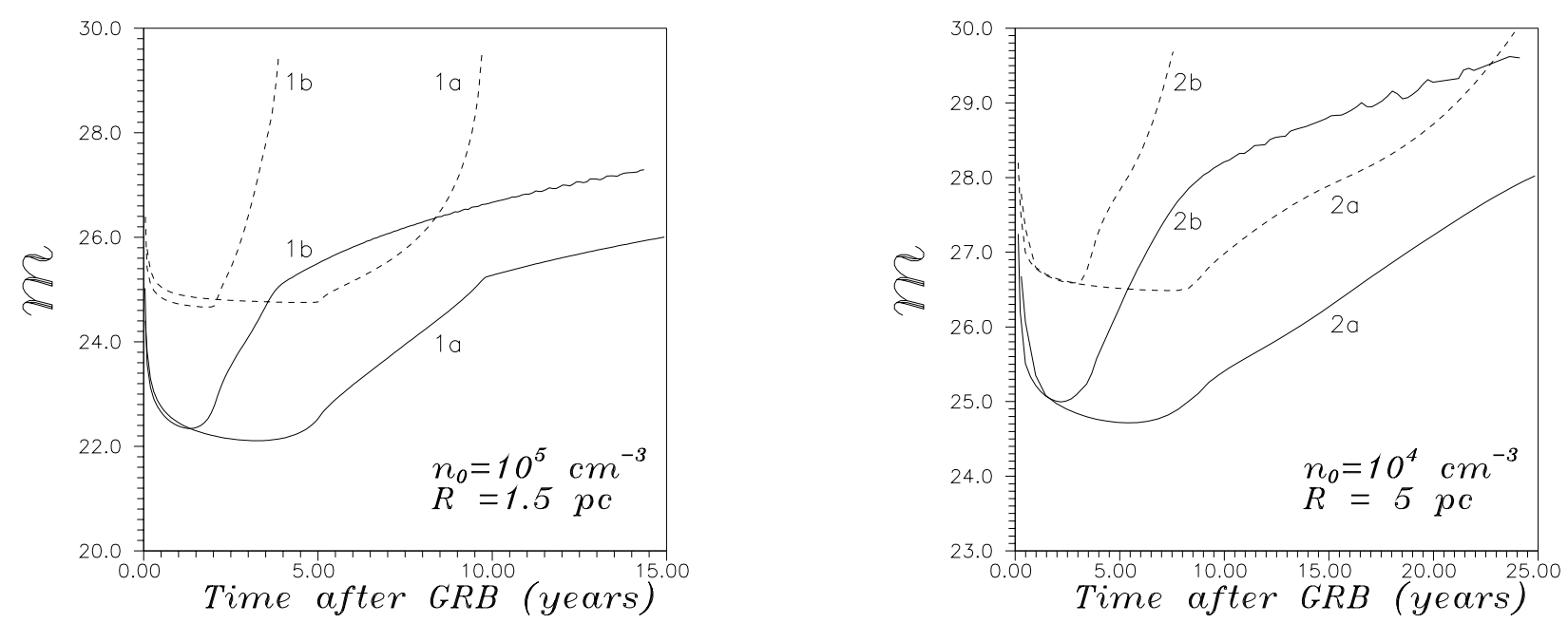

Fig. 1. The magnitudes of the counterparts (upper limit - solid line, lower limit - dashed line) as a function of time after burst for GRB with total flux near the Earth $F_{\mathrm{GRB}}=10^{-4} \mathrm{erg} \mathrm{cm}{ }^{-2}: 1 \mathrm{a}-$ for the case $E=10^{52} \mathrm{erg}, n_{0}=10^{5} \mathrm{~cm}^{-3} ; 1 \mathrm{~b}-$ for the case $E=10^{51} \mathrm{erg}, n_{0}=10^{5} \mathrm{~cm}^{-3} ; 2 \mathrm{a}$ - for the case $E=10^{52} \mathrm{erg}, n_{0}=10^{4} \mathrm{~cm}^{-3} ; 2 \mathrm{~b}$ - for the case $E=10^{51} \mathrm{erg}, n_{0}=10^{4} \mathrm{~cm}-3$

Electron density $\left(n_{\mathrm{e}}\right)$ is given by the Elvert formula.

This system was solved numerically using a full conservative difference scheme with flux corrected transport, because there is a strong density gradient in the solution (shock wave).

\section{Main results}

For GRB with the energy $10^{52}$ erg we considered the following densities of the surrounding interstellar medium: $10^{5} \mathrm{~cm}^{-3}, 300 \mathrm{~cm}^{-3}, 0.25 \mathrm{~cm}^{-3}, 1.610^{-3} \mathrm{~cm}^{-3}$. The main results of calculations in optically thin approximation are presented in our publications Timokhin \& BisnovatyiKogan (1995, 1996). According to these calculations, a counterpart of cosmological GRB due to the influence of GRB on the surrounding medium could be observed if density of the medium is greater then $300 \mathrm{~cm}^{-3}$. For such dense interstellar clouds optically thin approximation is not valid because of large optical depth in main emission lines.

Then we considered a simplified picture of conversion of UV photons into optical ones in optically thick regions, which allowed us to get optical and UV light curves of counterparts (see details in Bisnovatyi-Kogan \& Timokhin 1997). We considered GRBs with energies $10^{52}, 10^{51}$ ergs and following initial conditions in a gaseous cloud of radius $R$ :

$$
1 n_{0}=10^{5} \mathrm{~cm}^{-3}, T_{0}=20 \mathrm{~K}, R=1.5 \mathrm{pc}
$$$$
2 n_{0}=10^{4} \mathrm{~cm}^{-3}, T_{0}=20 \mathrm{~K}, R=5 \mathrm{pc} \text {. }
$$

The results of these calculations are presented in Fig. 1. Dashed lines give the lower boundary for luminosity, and solid lines - the upper one. For GRB with total energy output $10^{51}$ and $10^{52}$ ergs, maximal luminosities of counterparts differ by one order of magnitude too. This means that relative maximum brightness of the counterparts depends weakly on the total energy output. The total duration of the counterpart radiation decreases 2 times when the total output decreases 10 times. A more detailed description of the results is given in Bisnovatyi-Kogan \& Timokhin (1997).

\section{Conclusions}

It is shown, that counterparts of cosmological GRB due to interaction of gamma-radiation with dense interstellar media are "longliving" objects, existing for years after GRB. To distinguish GRB counterpart from a supernova event, having similar energy output, it is necessary to take into account its unusual light curve and spectrum. In the optical region of the spectrum the strongest emission lines are $\mathrm{H}_{\alpha}$ and $\mathrm{H}_{\beta}$.

The volume occupied by dense interstellar medium in the parent galaxy is relatively small, so the probability of getting a bright counterpart of this kind is also small. Nevertheless, discovery of even one optical counterpart of GRB with properties described above would give an opportunity to probe the density of the interstellar medium around the burst, and therefore would give an indication of the burst progenitor.

Acknowledgements. Part of this work was supported by Russian Foundation of Basic Research (RFFI) under grant 96-02-16553.

\section{References}

Bisnovatyi-Kogan G.S., Blinnikov S.I., 1980, MNRAS 191, 711 Cowie L., McKee C., Ostriker J., 1981, ApJ 247, 908

Timokhin A.N., Bisnovatyi-Kogan G.S., 1995, ApSS 231, 323 Timokhin A.N., Bisnovatyi-Kogan G.S., 1996, ApSS 235, 59 Bisnovatyi-Kogan G.S., Timokhin A.N., 1997, Astron. Reports 41, \#4, 423 